\title{
Mutation Analysis of the Common Deafness Genes in Patients with Nonsyndromic Hearing Loss in Linyi by SNPscan Assay
}

\author{
Fengguo Zhang, ${ }^{1,2}$ Yun Xiao, ${ }^{1,2}$ Lei Xu, ${ }^{1,2}$ Xue Zhang,, ${ }^{1,2}$ Guodong Zhang, \\ Jianfeng Li, ${ }^{1,2}$ Huaiqing Lv, ${ }^{3}$ Xiaohui Bai, ${ }^{1,2}$ and Haibo Wang ${ }^{1,2}$ \\ ${ }^{1}$ Department of Otorhinolaryngology Head and Neck Surgery, Shandong Provincial Hospital Affiliated to Shandong University, \\ Jinan 250022, China \\ ${ }^{2}$ Shandong Provincial Key Laboratory of Otology, Jinan 250022, China \\ ${ }^{3}$ Department of Otorhinolaryngology Head and Neck Surgery, People's Hospital of Linyi, Linyi, China
}

Correspondence should be addressed to Xiaohui Bai; sphbaixiaohui@hotmail.com and Haibo Wang; whbotologic797@163.com

Received 15 January 2016; Revised 6 April 2016; Accepted 17 April 2016

Academic Editor: Hideaki Moteki

Copyright (C) 2016 Fengguo Zhang et al. This is an open access article distributed under the Creative Commons Attribution License, which permits unrestricted use, distribution, and reproduction in any medium, provided the original work is properly cited.

\begin{abstract}
Hearing loss is a common sensory disorder, and at least $50 \%$ of cases are due to a genetic etiology. Although hundreds of genes have been reported to be associated with nonsyndromic hearing loss, GJB2, SLC26A4, and mtDNA12SrRNA are the major contributors. However, the mutation spectrum of these common deafness genes varies among different ethnic groups. The present work summarized mutations in these three genes and their prevalence in 339 patients with nonsyndromic hearing loss at three different special education schools and one children's hospital in Linyi, China. A new multiplex genetic screening system "SNPscan assay" was employed to detect a total of 115 mutations of the above three genes. Finally, $48.67 \%$ of the patients were identified with hereditary hearing loss caused by mutations in GJB2, SLC26A4, and mtDNA12SrRNA. The carrying rate of mutations in the three genes was $37.76 \%, 19.75 \%$, and $4.72 \%$, respectively. This mutation profile in our study is distinct from other parts of China, with high mutation rate of GJB2 suggesting a unique mutation spectrum in this area.
\end{abstract}

\section{Introduction}

Hearing loss or deafness is one of the most common neurosensory disorders in humans affecting about 1 to 3 in 1000 children worldwide [1]. It has been estimated that 30,000 newborns are detected with congenital hearing loss per 20 million live births every year in China [2], and approximately $50 \%$ of hearing loss is caused by a genetic etiology. At least $70 \%$ of all cases are classified as nonsyndromic hearing loss (NSHL) manifesting with isolated hearing loss without other associated clinical features. Of all NSHL, $75 \%-80 \%$ are autosomal recessive hereditary hearing loss, $15 \%-20 \%$ are autosomal dominant, $5 \%$ are X-linked, and $1 \%$ are inherited by mitochondrial genes [3].

Many previous genetic screening studies have shown that a large proportion of NSHL is caused by the mutations of a few genes, such as gap junction beta-2 protein (GJB2), solute carrier family 26 member 4 (SLC26A4), and mitochondrial DNA (mtDNA) 12SrRNA [4, 5]. Therefore, mutation screening of these common deafness-causing genes is of vital importance during genetic testing and counseling of deafness.

China harboring the highest population in the world is consisting of 56 nationalities. The mutation spectrum of the common deafness genes may vary among different ethnic nationalities due to geographical and language separation. The city Linyi with the biggest population and the largest area in Shandong Province locates in the east part of China. Almost all the populations in this area are Han nationality. People in Linyi are always impressed by introversion and rusticity. Because of its desolate geographical condition, Linyi city is a relatively conservative area with a less migration of 
population and an obstructive communication with other regions. Also, it has a less-developed economics for a long time. But to our knowledge, no systematic mutation analysis of deaf patients in Linyi has been reported previously. Therefore, there is a reason to believe that mutation spectrum of common deafness genes in patients with NSHL in this area must have a unique and special characteristic.

Up to now, many screening methods are used to detect the mutations of hearing loss, for example, direct sequencing, microarray, and PCR-restriction fragment length polymorphism (PCR-RFLP). These methods are either too expensive or time-consuming. Du et al. established a universal genetic screening system of hearing loss called the SNPscan assay which could detect 115 mutations of GJB2, $S L C 26 A 4$, and $m t D N A 12 S r R N A$ genes [6]. These selected mutations accounted for up to $90 \%$ of hearing loss caused by the most common deafness-causing genes in Chinese population.

In this study, we performed a comprehensive analysis of 3 common deafness-causing genes, GJB2, SLC26A4, and $m t D N A 12 S r R N A$, by SNPscan assay in 339 patients with NSHL from Linyi in eastern China. The aim was to investigate the molecular etiology in order to provide effective genetic counseling for hearing loss patients in this area.

\section{Materials and Methods}

2.1. Recruitment of the Subjects. A total of 339 subjects with NSHL from unrelated families were included in this study. They came from three different special education schools and one children's hospital in Linyi. Clinical questionnaire and informed consent were obtained from all subjects or their parents on their behalf. Clinical questionnaire showed basic information, including name, age, family history, health condition of the mother during pregnancy, and a clinical record of the patient, such as infections, possible head or brain injury, and the use of aminoglycoside antibiotics [7]. Physical and neurological examination was performed with special attention to renal, electrocardiac, and ophthalmologic differences to exclude those with syndromic hearing loss. An audiologic evaluation was performed including otoscope examination, tympanometry, and pure-tone audiometry (PTA). Auditory brainstem response (ABR) was employed in subjects when they were too young to accomplish the PTA test, and temporal bone computed tomography (CT) scan was also performed on participants for diagnosis of enlarged vestibular aqueduct (EVA) or inner ear malformation.

The onset of hearing impairment was categorized as prelingual/early ( $\leq 6$ years) and late ( $>6$ years). The degree of hearing impairment was calculated on the average of PTA in the speech frequencies $0.25,0.5,1,2$, and $4 \mathrm{kHz}$. Normal hearing was classified as PTA $\leq 25 \mathrm{~dB} \mathrm{nHL}$, mild hearing loss as PTA $>25 \mathrm{~dB} \mathrm{nHL}$ and $\leq 40 \mathrm{~dB} \mathrm{nHL}$, moderate hearing loss as PTA $>40 \mathrm{~dB} \mathrm{nHL}$ and $\leq 60 \mathrm{~dB} \mathrm{nHL}$, severe hearing loss as PTA $>60 \mathrm{~dB} \mathrm{nHL}$ and $\leq 80 \mathrm{~dB} \mathrm{nHL}$, and profound hearing loss as PTA $>80 \mathrm{~dB}$ nHL. To enroll in this study, all subjects should have bilateral, permanent sensorineural hearing impairment. This study was approved by the Ethics Committee of Shandong Provincial Hospital.

2.2. DNA Samples. Genomic DNA for all 339 patients was prepared from $2 \mathrm{~mL}$ of peripheral blood with AxyPrep Genomic Blood DNA Extraction Kit (AXYGEN, USA). For the evaluation of the quantity and quality of extracted DNA, spectrophotometry (UNICO 2100, USA) and 1\% agarose gel electrophoresis were carried out according to routine methods [8].

2.3. SNPscan for Mutation Detection. Two customized multiplex SNPscan assays from Genesky Biotechnologies Inc. (Shanghai, China) were designed to capture a total of 115 mutations of the three common deafness-causing genes as previously described (Table S1 in Supplementary Material available online at http://dx.doi.org/10.1155/2016/1302914): one is 53-plex and the other is 62-plex [6]. As a highthroughput and cost-saving SNP genotyping method, the SNPscan assay is based on double ligation and multiplex fluorescence PCR as described [9-11]. The SNPscan assays were done according to the detailed protocol described by $\mathrm{Du}$ et al. [6].

2.4. Statistical Analysis. The statistical analysis was performed using SAS 9.1.3 software (SAS, USA).

\section{Result}

3.1. Characterization of the Deaf Probands. A total of 339 probands with NSHL were recruited in this sequencing study, including 304 simplex and 35 multiplex probands. Simplex probands refer to the sporadic patients whose families have no one else suffering from hearing loss. Multiplex probands mean that there is at least one first- or second-degree deaf relative in the family. Clinical characterization of the deaf probands was summarized in Table 1. The patient cohort consisted of 196 males and 143 females, and they were all Han Chinese. The ages of the probands varied between a few months and 24 years (mean 8.9 years, 95\% CI 8.3-9.5 years). Only one proband had late-onset, progressive, and moderate sensorineural hearing loss. All other probands had prelingual or early-onset sensorineural hearing loss. Hearing tests demonstrated that the level of hearing loss was severe to profound in 328 patients. The remaining 11 patients showed moderate hearing loss.

Among all these patients, $48.67 \%$ were identified with hereditary hearing loss by means of the SNPscan assay (Figure 1). There were 104 patients (30.68\%) affected by GJB2 mutations: 49 homozygotes and 55 compound heterozygotes. 45 (13.27\%) patients carried two SLC26A4 pathogenic mutations: fourteen homozygotes and 31 compound heterozygotes. $16(4.72 \%)$ patients harbored mtDNA12SrRNA mutations consisting of 15 homoplasmic mutations and 1 heteroplasmic mutation. 
TABLE 1: Deaf patients $(n=339)$ categorized by their clinical characteristics.

\begin{tabular}{lcc}
\hline & $\begin{array}{c}\text { Simplex } \\
(n=304)\end{array}$ & $\begin{array}{c}\text { Multiplex } \\
(n=35)\end{array}$ \\
\hline Sex & 179 & 17 \\
$\quad$ Male & 125 & 18 \\
$\quad$ Female & & \\
Age at the test & 153 & 13 \\
0-6 years & 122 & 21 \\
6-18 years & 29 & 1 \\
18-35 years & & \\
Age of onset & 303 & 35 \\
$\quad$ Early onset ( $\leq 6$ years) & 1 & 0 \\
Late onset (>6 years) & & \\
Severity of hearing impairment & 0 & 0 \\
$\quad$ Mild & 11 & 5 \\
$\quad$ Moderate & 56 & 30 \\
$\quad$ Severe & 237 & \\
Profound & &
\end{tabular}
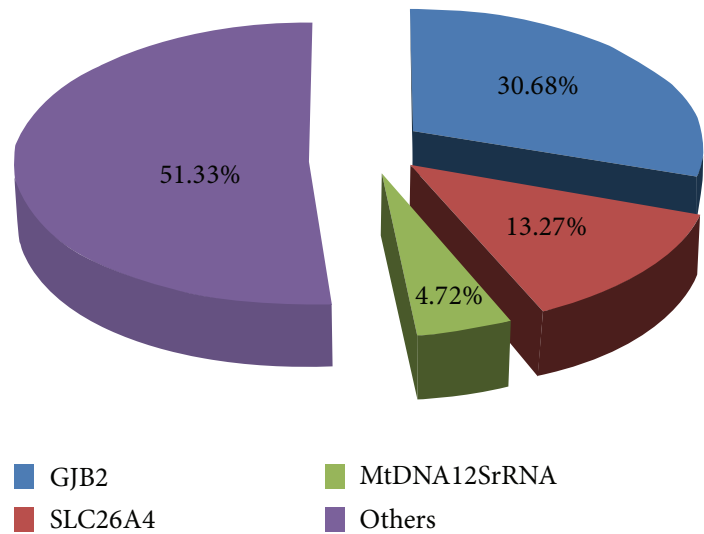

MtDNA12SrRNA

SLC26A4

Others

FIGURE 1: Distribution of the common deafness-causing genes in 339 NSHL patients.

3.2. Mutations in GJB2 Gene. Eleven variants were identified in this cohort. They were three frameshift deletions (c.176_191del16, c.235delC, and c.299_300delAT), two frameshift insertions (c.34_35insG and c.511_512insAACG), four missense mutations [c.109G $>$ A (p.V37I), c.257C>G (p.T86R), c.427C > T (p.R143W), and c.571T>C (p.F191L)], one nonsense mutation c.9G $>$ A (p.W3X), and one splicing site mutation IVS1+1G $>$ A (shown in Table 2). Nine of them were pathological mutations which have been determined previously. The category of two nucleotide changes was unknown (c.9G>A, c.571T>C). The mutant alleles of GJB2 accounted for $34.37 \%(233 / 678)$ of the total alleles in all patients (Table 3 ). The most common mutation allele of GJB2 in this area was c.235delC with a mutant frequency of $20.65 \%$ (140/678). The second common was c.299_300delAT of $5.60 \%$ (38/678), followed by c.109G $>$ A of $2.51 \%(17 / 678)$, c.34_35insG of $1.77 \%$ (12/678), c.176_191del16 of $1.33 \%$ (9/678),
c.511_512insAACG of $1.18 \%(8 / 678)$, c. $257 \mathrm{C}>\mathrm{G}$ of $0.29 \%$ $(2 / 678)$, c. $427 \mathrm{C}>\mathrm{T}$ of $0.29 \%(2 / 678)$, and IVS1+1G $>\mathrm{A}$ of $0.15 \%$ (1/678) (1/678) (Table 3).

A total of 104 patients (30.68\%) were confirmed to be associated with hereditary hearing loss caused by GJB2 mutation: 49 homozygotes ( 42 with the c.235delC allele, 4 with c.299_300delAT, and 3 with the c.109G $>$ A allele) and 55 compound heterozygotes. Twenty patients $(5.90 \%)$ had monoallelic variants in the heterozygous form: ten with c.235delC, eight with c.109G >A, one with c.299_300delAT, and one with c.511_512insAACG. Totally, $128(37.76 \%, 128 / 339)$ patients had molecular defects in GJB2 gene including three patients with c.571T $>$ C mutation (Table 2).

The most common mutation allele of GJB2 was c. $235 \mathrm{delC}$ with a mutant frequency of 20.65\% (140/678) affecting 98 patients. Among these patients carrying at least one c. $235 \mathrm{delC}$ allele, there were 42 homozygotes, 46 compound heterozygotes, and 10 single heterozygotes.

3.3. Mutations in SLC26A4 Gene. Nineteen variants were identified in this cohort, including 12 missense mutations [c.147C > G (p.S49R), c.269C >T (p.S90L), c.563T $>C$ (p.I188T), c.589G>A (p.G197R), c.1173C >A (p.S391R), c.1174A $>$ T (p.N392Y), c.1225C $>$ T (p.R409C), c.1226G $>$ A (p.R409H), c.1975G >C (p.V659L), c.1985G>A (p.C662Y), c.2027T $>$ A (p.L676Q), and c.2168A $>$ G (p.H723R)], four nonsense mutations [c.235C $>\mathrm{T}$ (p.R79X), c.946G $>\mathrm{T}$ (p.G316X), c.1318A > T (p.K440X), and c.1540C>T (p.Q514X)], two splicing site mutations (c.919-2A>G, c.1707+5 G>A), and one frameshift insertion mutation (c.1547_1548insC) (Table 5). Combining the prediction of SIFT and Polyphen-2 with the results of previous studies, all variants analyzed in our cohort were considered pathogenic mutations except c.147C>G (p.S49R) for an unknown significant pathogenicity (Table 4 ).

There were $45(13.27 \%)$ patients who were confirmed to have inherited hearing loss because they carried two SLC26A4 pathogenic mutations: fourteen homozygotes (13 c. IVS $7-2 A>G$ and 1 c. $2168 \mathrm{~A}>\mathrm{G}$ ) and 31 compound heterozygotes. And 22 (6.49\%) patients who were identified carried one SLC26A4 pathogenic mutation. Thus, the detection rate of SLC26A4 mutations was $19.76 \%(67 / 339)$ in this patient cohort. All patients carrying two mutations were diagnosed with EVA syndrome by CT scan of the temporal bone. And 17 out of 22 patients carrying one pathogenic mutation were diagnosed with EVA syndrome.

The most common mutation allele of SLC26A4 was c.919$2 \mathrm{~A}>\mathrm{G}$ with a mutant frequency of $9.59 \%$ (65/678) affecting 52 patients. Among these patients carrying at least one c.919-2A>G mutation allele, there were 13 homozygotes, 27 compound heterozygotes, and 12 single heterozygotes. The second common mutation allele of SLC26A4 was c. $2168 \mathrm{~A}>\mathrm{G}$ with a mutant frequency of $2.21 \%(15 / 678)$.

3.4. Mutations in mtDNA12SrRNA. Sixteen patients $(4.72 \%)$ carried an mtDNA12SrRNA mutation, where all of which were the m.1555A $>\mathrm{G}$ mutation containing 15 homoplasmic 
TABLE 2: GJB2 genotypes of deaf patients from Linyi.

\begin{tabular}{|c|c|c|c|c|c|c|}
\hline & Allele 1 & & & Allele 2 & & \\
\hline $\begin{array}{l}\text { Nucleotide } \\
\text { change }\end{array}$ & $\begin{array}{c}\text { Consequence } \\
\text { or amino acid } \\
\text { change }\end{array}$ & Category & $\begin{array}{l}\text { Nucleotide } \\
\text { change }\end{array}$ & $\begin{array}{c}\text { Consequence } \\
\text { or amino acid } \\
\text { change }\end{array}$ & Category & Number of patients \\
\hline $\mathrm{IVS} 1+1 \mathrm{G}>\mathrm{A}$ & Splicing site & Pathogenic & c. $9 \mathrm{G}>\mathrm{A}$ & p.W3X & Unknown & 1 \\
\hline 34_35insG & Frameshift & Pathogenic & $109 \mathrm{G}>\mathrm{A}$ & p.V37I & Pathogenic & 1 \\
\hline 34_35insG & Frameshift & Pathogenic & 176_191del16 & Frameshift & Pathogenic & 1 \\
\hline 34_35insG & Frameshift & Pathogenic & 235delC & Frameshift & Pathogenic & 8 \\
\hline 34_35insG & Frameshift & Pathogenic & 299_300delAT & Frameshift & Pathogenic & 2 \\
\hline $109 \mathrm{G}>\mathrm{A}$ & p.V37I & Pathogenic & $109 \mathrm{G}>\mathrm{A}$ & p.V37I & Pathogenic & 3 \\
\hline $109 \mathrm{G}>\mathrm{A}$ & p.V37I & Pathogenic & $235 \mathrm{delC}$ & Frameshift & Pathogenic & 1 \\
\hline $109 \mathrm{G}>\mathrm{A}$ & p.V37I & Pathogenic & 299_300delAT & Frameshift & Pathogenic & 1 \\
\hline $109 \mathrm{G}>\mathrm{A}$ & p.V37I & Pathogenic & - & & & 8 \\
\hline 176_191del16 & Frameshift & Pathogenic & 235delC & Frameshift & Pathogenic & 8 \\
\hline 235delC & Frameshift & Pathogenic & 235delC & Frameshift & Pathogenic & 42 \\
\hline $235 \mathrm{delC}$ & Frameshift & Pathogenic & $257 \mathrm{C}>\mathrm{G}$ & p.T86R & Pathogenic & 2 \\
\hline 235delC & Frameshift & Pathogenic & 299_300delAT & Frameshift & Pathogenic & 22 \\
\hline 235delC & Frameshift & Pathogenic & $427 \mathrm{C}>\mathrm{T}$ & p.R143W & Pathogenic & 2 \\
\hline 235delC & Frameshift & Pathogenic & 511_512insAACG & Frameshift & Pathogenic & 3 \\
\hline $235 \mathrm{delC}$ & Frameshift & Pathogenic & - & & & 10 \\
\hline 299_300delAT & Frameshift & Pathogenic & 299_300delAT & Frameshift & Pathogenic & 4 \\
\hline 299_300delAT & Frameshift & Pathogenic & 511_512insAACG & Frameshift & Pathogenic & 4 \\
\hline 299_300delAT & Frameshift & Pathogenic & - & & & 1 \\
\hline 511_512insAACG & Frameshift & Pathogenic & - & & & 1 \\
\hline $571 \mathrm{~T}>\mathrm{C}$ & p.F191L & Unknown & - & & & 3 \\
\hline
\end{tabular}

TABLE 3: Allele frequencies of GJB2 mutations in 339 deaf patients from Linyi.

\begin{tabular}{lccc}
\hline Mutations & Consequence & $\begin{array}{c}\text { Number of } \\
\text { alleles }\end{array}$ & $\begin{array}{c}\text { Allele } \\
\text { frequency (\%) }\end{array}$ \\
\hline IVS1+1G>A & Splice site & 1 & 0.15 \\
c.34_35insG & Frameshift & 12 & 1.77 \\
c.109G>A & p.V37I & 17 & 2.51 \\
c.176_191del16 & Frameshift & 9 & 1.33 \\
c.235delC & Frameshift & 140 & 20.65 \\
c.257C $>$ G & p.T86R & 2 & 0.29 \\
c.299_300delAT & Frameshift & 38 & 5.60 \\
c.427C>T & p.R143W & 2 & 0.29 \\
c.511_512insAACG & Frameshift & 8 & 1.18 \\
\hline
\end{tabular}

mutations and 1 heteroplasmic mutations. Ten of these patients had a clear history of aminoglycoside antibiotic use.

\section{Discussion}

Currently, many studies have reported that GJB2, SLC26A4, and $m t D N A 12 S r R N A$ genes are the most common causes in Chinese NSHL population [12-14]. In the present study we have screened for the common deafness gene mutations of 339 NSHL children from Linyi, east part of China. SNPscan assay was used to perform genotyping detection of these three common deafness-causing genes. Compared with other methods, this technique is accurate, rapid, and economically effective. As the novel mutations have been reported successively, more mutation alleles should be added in the SNPscan assay.

4.1. GJB2 Mutation Analysis. In this present study, GJB2 mutations were detected in $37.76 \%$ (128/339) of all patients, including $30.68 \%$ (104/339) with two pathogenic mutations and $7.08 \%(24 / 339)$ with only one mutant allele. The c. $235 \mathrm{delC}$ and c.299_300delAT were the most frequent mutations in the NSHL patients in Linyi, whereas the hotspot mutation in Xiamen, China, was c.109G >A [7].

The frameshift mutation c. $235 \mathrm{delC}$ has been reported as the most common mutation causing premature protein termination in hearing impaired patients in East and Southeast Asia, while lower frequencies were reported in Europe and Oceania [15-24]. The c.235delC mutation allele frequency was $20.65 \%(140 / 678)$ in this work. In an earlier nationwide study, Dai et al. analyzed the GJB2 mutation of 2063 unrelated NSHL students from 23 different regions of China, and the c. 235 delC mutation allele frequency was $12.34 \%(509 / 4,126)$ 
TABLE 4: SLC26A4 genotypes of deaf patients from Linyi.

\begin{tabular}{|c|c|c|c|c|c|c|}
\hline & Allele 1 & & & Allele 2 & & \\
\hline $\begin{array}{l}\text { Nucleotide } \\
\text { change }\end{array}$ & $\begin{array}{c}\text { Consequence } \\
\text { or amino acid } \\
\text { change }\end{array}$ & Category & $\begin{array}{l}\text { Nucleotide } \\
\text { change }\end{array}$ & $\begin{array}{c}\text { Consequence } \\
\text { or amino acid } \\
\text { change }\end{array}$ & Category & Number of patients \\
\hline c. $147 \mathrm{C}>\mathrm{G}$ & p.S49R & Unknown & - & & & 1 \\
\hline c. $235 \mathrm{C}>\mathrm{T}$ & p.R79X & Pathogenic & c. $919-2 A>G$ & Splice site & Pathogenic & 1 \\
\hline c. $235 \mathrm{C}>\mathrm{T}$ & p.R79X & Pathogenic & - & & & 1 \\
\hline c. $269 \mathrm{C}>\mathrm{T}$ & p.S90L & Pathogenic & - & & & 1 \\
\hline c. $563 \mathrm{~T}>\mathrm{C}$ & p.I188T & Pathogenic & c. $919-2 A>G$ & Splice site & Pathogenic & 1 \\
\hline c. $589 \mathrm{G}>\mathrm{A}$ & p.G197R & Pathogenic & c. $919-2 A>G$ & Splice site & Pathogenic & 1 \\
\hline c. $919-2 A>G$ & Splice site & Pathogenic & c. $919-2 A>G$ & Splice site & Pathogenic & 13 \\
\hline c. $919-2 A>G$ & Splice site & Pathogenic & c. $946 \mathrm{G}>\mathrm{T}$ & p.G316X & Pathogenic & 1 \\
\hline c. $919-2 A>G$ & Splice site & Pathogenic & c. $1174 \mathrm{~A}>\mathrm{T}$ & p.N392Y & Pathogenic & 2 \\
\hline c. $919-2 A>G$ & Splice site & Pathogenic & c. $1226 \mathrm{G}>\mathrm{A}$ & p.R409H & Pathogenic & 3 \\
\hline c. $919-2 A>G$ & Splice site & Pathogenic & c. $1318 \mathrm{~A}>\mathrm{T}$ & p.K440X & Pathogenic & 1 \\
\hline c. $919-2 A>G$ & Splice site & Pathogenic & c. $1540 \mathrm{C}>\mathrm{T}$ & p.Q514X & Pathogenic & 1 \\
\hline c. $919-2 A>G$ & Splice site & Pathogenic & c.1547_1548InsC & Frameshift & Pathogenic & 1 \\
\hline c. $919-2 A>G$ & Splice site & Pathogenic & c. $1707+5 \mathrm{G}>\mathrm{A}$ & Splice site & Pathogenic & 3 \\
\hline c. $919-2 A>G$ & Splice site & Pathogenic & c. $1975 \mathrm{G}>\mathrm{C}$ & p.V659L & Pathogenic & 3 \\
\hline c. $919-2 A>G$ & Splice site & Pathogenic & c. $1985 \mathrm{G}>\mathrm{A}$ & p.C662Y & Pathogenic & 1 \\
\hline c. $919-2 A>G$ & Splice site & Pathogenic & c. $2027 \mathrm{~T}>\mathrm{A}$ & p.L676Q & Pathogenic & 2 \\
\hline c. $919-2 A>G$ & Splice site & Pathogenic & c. $2168 \mathrm{~A}>\mathrm{G}$ & p.H723R & Pathogenic & 6 \\
\hline c. $919-2 A>G$ & Splice site & Pathogenic & - & & & 12 \\
\hline c. $946 \mathrm{G}>\mathrm{T}$ & p.G316X & Pathogenic & c. $2168 \mathrm{~A}>\mathrm{G}$ & p.H723R & Pathogenic & 1 \\
\hline c. $1173 \mathrm{C}>\mathrm{A}$ & p.S391R & Pathogenic & c. $2168 \mathrm{~A}>\mathrm{G}$ & p.H723R & Pathogenic & 1 \\
\hline c. $1225 \mathrm{C}>\mathrm{T}$ & p.R409C & Pathogenic & - & & & 1 \\
\hline c. $1226 \mathrm{G}>\mathrm{A}$ & p.R409H & Pathogenic & c. $1975 \mathrm{G}>\mathrm{C}$ & p.V659L & Pathogenic & 1 \\
\hline c. $1226 \mathrm{G}>\mathrm{A}$ & p.R409H & Pathogenic & c. $2168 \mathrm{~A}>\mathrm{G}$ & p.H723R & Pathogenic & 1 \\
\hline c. $1226 \mathrm{G}>\mathrm{A}$ & p.R409H & Pathogenic & - & & & 1 \\
\hline c.1975G $>C$ & p.V659L & Pathogenic & - & & & 1 \\
\hline c. $2168 \mathrm{~A}>\mathrm{G}$ & p.H723R & Pathogenic & c. $2168 \mathrm{~A}>\mathrm{G}$ & p.H723R & Pathogenic & 1 \\
\hline c. $2168 \mathrm{~A}>\mathrm{G}$ & p.H723R & Pathogenic & - & & & 4 \\
\hline
\end{tabular}

[25]. Compared with $12.34 \%$ in his study, the difference in the c.235delC mutation allele frequency was significant $(P=0.001)$, reflecting a certain distinction of the c.235delC mutation frequency in Linyi and other areas. There was a higher carrying rate of c. $235 \mathrm{delC}$ in the Linyi deaf population.

4.2. SLC26A4 Mutation Analysis. According to previous study, more than 200 mutations have been described in the SLC26A4 gene with Pendred syndrome (PS) and enlarged vestibular aqueduct (EVA) syndrome (http://www.healthcare .uiowa.edu/labs/pendredandbor/) showing specific distinctions among racial backgrounds. Most of them are missense mutations, in addition to frameshift mutations, splice site mutations, insertions, or deletions [26].

The mutation hotspots of SLC26A4 differed among different nations and areas. In the present study, the most common mutation in our patient cohort was c.IVS7-2A>G and the mutation allele frequency was $9.59 \%$ (65/678), whereas the mutation hot spots are p.T416P and c.IVS8+1G $>$ A [27] in Northern Europe, p.H723R and c. IVS7-2A $>$ G in South Korea [15], and p.H723R in Japan [28]. It was reported in a mutation study of a large cohort in Chinese deaf population that the most common SLC26A4 mutation was also IVS7$2 \mathrm{~A}>\mathrm{G}(8.65 \%, 566 / 6,542)[12]$. Although the mutation allele frequency was a little higher $(9.59 \%)$ in our study, these frequencies of c.IVS7-2A $>\mathrm{G}$ are not significantly different $(P$ $=0.41)$.

4.3. $m$ tDNA12SrRNA Mutation Analysis. Patients with mutations in $m t D N A 12 S r R N A$ can be affected with aminoglycoside antibiotic-induced deafness showing a hereditary model of maternal inheritance. And m.1494C $>\mathrm{T}$ and $\mathrm{m} .1555 \mathrm{~A}>\mathrm{G}$ are the most common mutations of this mitochondrial gene. Mutate rate of this gene varies among racial and geographic 
TABLE 5: Allele frequencies of SLC26A4 mutations in 339 deaf patients from Linyi.

\begin{tabular}{lccc}
\hline Mutations & Consequence & $\begin{array}{c}\text { Number of } \\
\text { alleles }\end{array}$ & $\begin{array}{c}\text { Allele } \\
\text { frequency } \\
(\%)\end{array}$ \\
\hline c.235C $>$ T & p.R79X & 2 & 0.29 \\
c.269C $>$ T & p.S90L & 1 & 0.15 \\
c.563T $>$ C & p.I188T & 1 & 0.15 \\
c.589G $>$ A & p.G197R & 1 & 0.15 \\
c.919-2A $>$ G & Splice site & 65 & 9.59 \\
c.946G $>$ T & p.G316X & 2 & 0.29 \\
c.1173C $>$ A & p.S391R & 1 & 0.15 \\
c.1174A $>$ T & p.N392Y & 2 & 0.29 \\
c.1225C $>$ T & p.R409C & 1 & 0.15 \\
c.1226G $>$ A & p.R409H & 6 & 0.88 \\
c.1318A $>$ T & p.K440X & 1 & 0.15 \\
c.1540C $>$ T & p.Q514X & 1 & 0.15 \\
c.1547_1548InsC & Frameshift & 1 & 0.15 \\
c.1707+5 G $>$ A & Splicing site & 3 & 0.44 \\
c.1975G $>$ C & p.V659L & 5 & 0.74 \\
c.1985G $>$ A & p.C662Y & 1 & 0.15 \\
c.2027T $>$ A & p.L676Q & 2 & 0.29 \\
c.2168A $>$ G & p.H723R & 15 & 2.21 \\
\hline & & &
\end{tabular}

origins in populations with NSHL, with a frequency of $0.6-2.5 \%$ in Caucasians [29-32], $1.8 \%$ in Turks [30], 0.7\% in Germans, $1.8 \%$ in Hungarians, and $2.4 \%$ in Poles [29]. In our study, we found that $4.72 \%$ of the patients were identified to have NSHL caused by the m.1555A>G mutation of $m t D N A 12 S r R N A$ gene which was close to that observed by Dai et al. $(3.87 \%)(P=0.53)$.

In summary, we performed the first genetic analysis of hearing loss patients from Linyi, eastern part of China. According to genetic detections of GJB2, SLC26A4, and $m t D N A 12 S r R N A$ via the SNPscan assay, the fact that almost half of the patients with nonsyndromic hearing loss appeared to have a genetic etiology shows that the SNPscan assay is an available diagnosis tool for studies on genetic hearing loss. The results of the detection suggest that GJB2 mutations appear to be a major cause of congenital hearing loss in Linyi. The prevalence of SLC26A4 mutations and $m t D N A 12 S r R N A$ mutations was very close to other previous studies in Chinese deaf population. However, about $50 \%$ of NSHL patients are still not identified with a molecular etiology. For this reason, copy number variations (CNVs) of the three common deafness-causing genes need to be detected. Also, next generation sequencing (NGS) will be employed in screening other deafness-causing genes in the future.

\section{Conclusions}

In this study, we used the SNPscan assay technique to detect the 115 mutations of the three common deafness-causing genes in 339 nonsyndromic hearing loss patients from Linyi,
China. And we found that the mutation profile was distinct from other parts of China suggesting a unique mutation spectrum in this area. Also, the SNPscan assay was an available diagnosis tool for studies on genetic hearing loss.

\section{Competing Interests}

The authors declare that they have no competing interests.

\section{Acknowledgments}

This work was supported by grants from the National 973 Basic Research Program of China (2014CB541703), the Research Special Fund for Public Welfare Industry of Health (201202001), grants from the National Natural Science Foundation of China (81470693 and 81470704), a grant from the Project funded by China Postdoctoral Science Foundation (2014M560563 and 2015T80726), and grants from the Natural Science Foundation of Shandong Province (ZR2014HM022 and ZR2014HP064). The authors sincerely thank all the family members for their participation and cooperation in this study.

\section{References}

[1] A. L. Mehl and V. Thomson, "The Colorado newborn hearing screening project, 1992-1999: on the threshold of effective population-based universal newborn hearing screening," Pediatrics, vol. 109, no. 1, 2002.

[2] P. Dai, X. Liu, D. Han et al., "Extremely low penetrance of deafness associated with the mitochondrial $12 \mathrm{~S}$ rRNA mutation in 16 Chinese families: implication for early detection and prevention of deafness," Biochemical and Biophysical Research Communications, vol. 340, no. 1, pp. 194-199, 2006.

[3] S. T. Bozdogan, G. Kuran, Ö. Ö. Yüregir et al., "The prevalence of Gap Junction Protein Beta 2 (GJB2) mutations in non syndromic sensorineural hearing loss in çukurova region," Journal of International Advanced Otology, vol. 11, no. 2, pp. 118121, 2015.

[4] Y. Yuan, Y. You, D. Huang et al., "Comprehensive molecular etiology analysis of nonsyndromic hearing impairment from typical areas in China," Journal of Translational Medicine, vol. 7, article 79, 2009.

[5] F. Xin, Y. Yuan, X. Deng et al., "Genetic mutations in nonsyndromic deafness patients of Chinese minority and han ethnicities in Yunnan, China," Journal of Translational Medicine, vol. 11, article 312, 2013.

[6] W. Du, J. Cheng, H. Ding, Z. Jiang, Y. Guo, and H. Yuan, "A rapid method for simultaneous multi-gene mutation screening in children with nonsyndromic hearing loss," Genomics, vol. 104, no. 4, pp. 264-270, 2014.

[7] Y. Jiang, S. Huang, T. Deng et al., "Mutation spectrum of common deafness-causing genes in patients with non-syndromic deafness in the Xiamen Area, China," PLoS ONE, vol. 10, no. 8, Article ID e0135088, 2015.

[8] X. Bai, H. Lv, F. Zhang et al., "Identification of a novel missense mutation in the WFS1 gene as a cause of autosomal dominant nonsyndromic sensorineural hearing loss in all-frequencies," American Journal of Medical Genetics Part A, vol. 164, no. 12, pp. 3052-3060, 2014. 
[9] X. Chen, S. Li, Y. Yang et al., "Genome-wide association study validation identifies novel loci for atherosclerotic cardiovascular disease," Journal of Thrombosis and Haemostasis, vol. 10, no. 8, pp. 1508-1514, 2012.

[10] J. Wei, L. Zheng, S. Liu et al., "MiR-196a2 rs11614913 T>C polymorphism and risk of esophageal cancer in a Chinese population," Human Immunology, vol. 74, pp. 1199-1205, 2013.

[11] L. Zheng, J. Yin, L. Wang et al., "Interleukin 1B rs16944 G>A polymorphism was associated with a decreased risk of esophageal cancer in a Chinese population," Clinical Biochemistry, vol. 46, no. 15, pp. 1469-1473, 2013.

[12] P. Dai, Q. Li, D. Huang et al., "SLC26A4 c.919-2A>G varies among Chinese ethnic groups as a cause of hearing loss," Genetics in Medicine, vol. 10, no. 8, pp. 586-592, 2008.

[13] S.-H. Duan, Y.-M. Zhu, Y.-L. Wang, and Y.-F. Guo, "Common molecular etiology of nonsyndromic hearing loss in 484 patients of 3 ethnicities in northwest China," Acta OtoLaryngologica, vol. 135, no. 6, pp. 586-591, 2015.

[14] Y. Fang, M. Gu, C. Wang, F. Suo, G. Wang, and Y. Xia, “ GJB2 as well as SLC26A4 gene mutations are prominent causes for congenital deafness," Cell Biochemistry and Biophysics, vol. 73, no. 1, pp. 41-44, 2015.

[15] K. Tsukamoto, H. Suzuki, D. Harada et al., "Distribution and frequencies of PDS (SLC26A4) mutations in Pendred syndrome and nonsyndromic lossassociated with enlarged vestibular aqueduct: a unique spectrum of mutations in Japanese," European Journal of Human Genetics, vol. 11, no. 12, pp. 916-922, 2003.

[16] C.-C. Lai, C.-Y. Chiu, A.-S. Shiao et al., "Analysis of the SLC26A4 gene in patients with Pendred syndrome in Taiwan," Metabolism: Clinical and Experimental, vol. 56, no. 9, pp. 12791284, 2007.

[17] S. Huang, D. Han, Y. Yuan et al., "Extremely discrepant mutation spectrum of SLC26A4 between Chinese patients with isolated Mondini deformity and enlarged vestibular aqueduct," Journal of Translational Medicine, vol. 9, article 167, 2011.

[18] Y. Okamoto, H. Mutai, A. Nakano et al., "Subgroups of enlarged vestibular aqueduct in relation to SLC26A4 mutations and hearing loss," Laryngoscope, vol. 124, no. 4, pp. E134-E140, 2014.

[19] H. Hu, L. Wu, Y. Feng et al., "Molecular analysis of hearing loss associated with enlarged vestibular aqueduct in the mainland Chinese: a unique SLC26A4 mutation spectrum," Journal of Human Genetics, vol. 52, no. 6, pp. 492-497, 2007.

[20] J.-J. Yang, C.-C. Tsai, H.-M. Hsu, J.-Y. Shiao, C.-C. Su, and S.-Y. $\mathrm{Li}$, "Hearing loss associated with enlarged vestibular aqueduct and Mondini dysplasia is caused by splice-site mutation in the PDS gene," Hearing Research, vol. 199, no. 1-2, pp. 22-30, 2005.

[21] B. Y. Choi, A. C. Madeo, K. A. King et al., "Segregation of enlarged vestibular aqueducts in families with non-diagnostic SLC26A4 genotypes," Journal of Medical Genetics, vol. 46, no. 12, pp. 856-861, 2009.

[22] S.-Y. Nishio and S.-I. Usami, "Deafness gene variations in a 1120 nonsyndromic hearing loss cohort: molecular epidemiology and deafness mutation spectrum of patients in Japan," The Annals of Otology Rhinology and Laryngology, vol. 124, no. 1, supplement, pp. 49S-60S, 2015.

[23] D. Wattanasirichaigoon, C. Limwongse, C. Jariengprasert et al., "High prevalence of V37I genetic variant in the connexin26 (GJB2) gene among non-syndromic hearing-impaired and control Thai individuals," Clinical Genetics, vol. 66, no. 5, pp. 452-460, 2004.
[24] S.-H. Han, H.-J. Park, E.-J. Kang et al., "Carrier frequency of GJB2 (connexin-26) mutations causing inherited deafness in the Korean population," Journal of Human Genetics, vol. 53, no. 11-12, pp. 1022-1028, 2008.

[25] P. Dai, F. Yu, B. Han et al., "GJB2 mutation spectrum in 2,063 Chinese patients with nonsyndromic hearing impairment," Journal of Translational Medicine, vol. 7, article 26, 2009.

[26] S. Dossena, A. Bizhanova, C. Nofziger et al., "Identification of allelic variants of pendrin (SLC26A4) with loss and gain of function," Cellular Physiology and Biochemistry, vol. 28, no. 3, pp. 467-476, 2011.

[27] C. Campbell, R. A. Cucci, S. Prasad et al., "Pendred syndrome, DFNB4, and PDS/SLC26A4 identification of eight novel mutations and possible genotype-phenotype correlations," Human Mutation, vol. 17, no. 5, pp. 403-411, 2001.

[28] H.-J. Park, S.-J. Lee, H.-S. Jin et al., "Genetic basis of hearing loss associated with enlarged vestibular aqueducts in Koreans," Clinical Genetics, vol. 67, no. 2, pp. 160-165, 2005.

[29] S. Kupka, T. Tóth, M. Wróbel et al., "Mutation A1555G in the $12 \mathrm{~S}$ rRNA gene and its epidemiological importance in German, Hungarian, and Polish patients," Human Mutation, vol. 19, no. 3, pp. 308-309, 2002.

[30] M. Tekin, T. Duman, G. Boğoçlu et al., "Frequency of $m t D N A$ A1555G and A7445G mutations among children with prelingual deafness in Turkey," European Journal of Pediatrics, vol. 162, no. 3, pp. 154-158, 2003.

[31] R. Li, J. H. Greinwald Jr., L. Yang, D. I. Choo, R. J. Wenstrup, and M. X. Guan, "Molecular analysis of the mitochondrial $12 \mathrm{~S}$ rRNA and tRNASer(UCN) genes in paediatric subjects with non-syndromic hearing loss," Journal of Medical Genetics, vol. 41, no. 8, pp. 615-620, 2004.

[32] H. T. Jacobs, T. P. Hutchin, T. Käppi et al., "Mitochondrial DNA mutations in patients with postlingual, nonsyndromic hearing impairment," European Journal of Human Genetics, vol. 13, no. 1, pp. 26-33, 2005. 


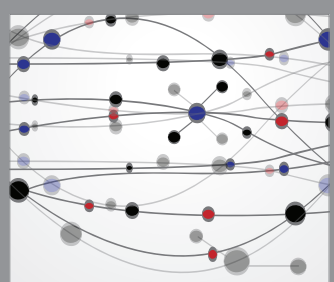

The Scientific World Journal
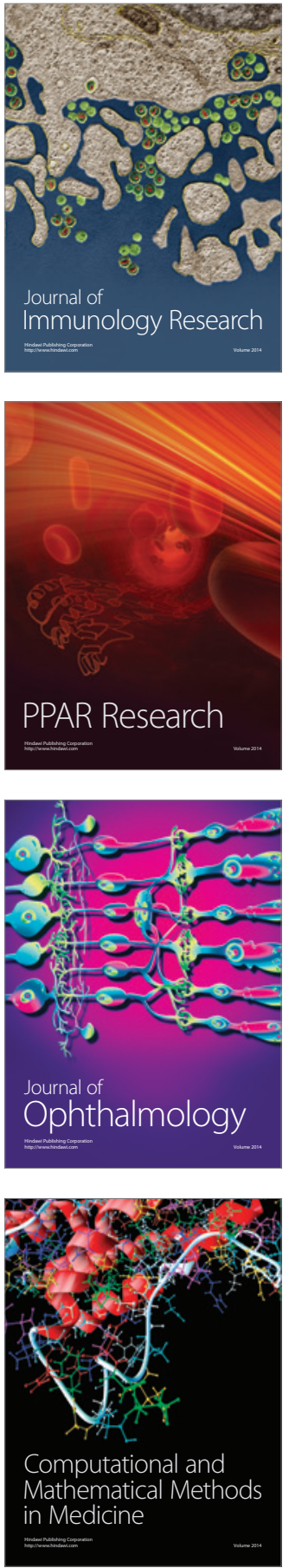

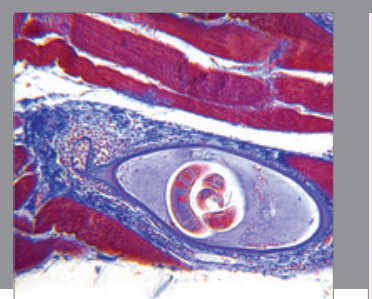

Gastroenterology Research and Practice

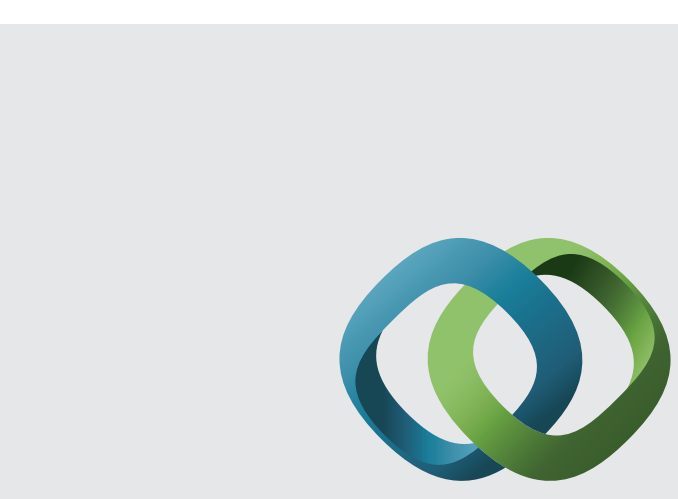

\section{Hindawi}

Submit your manuscripts at

http://www.hindawi.com
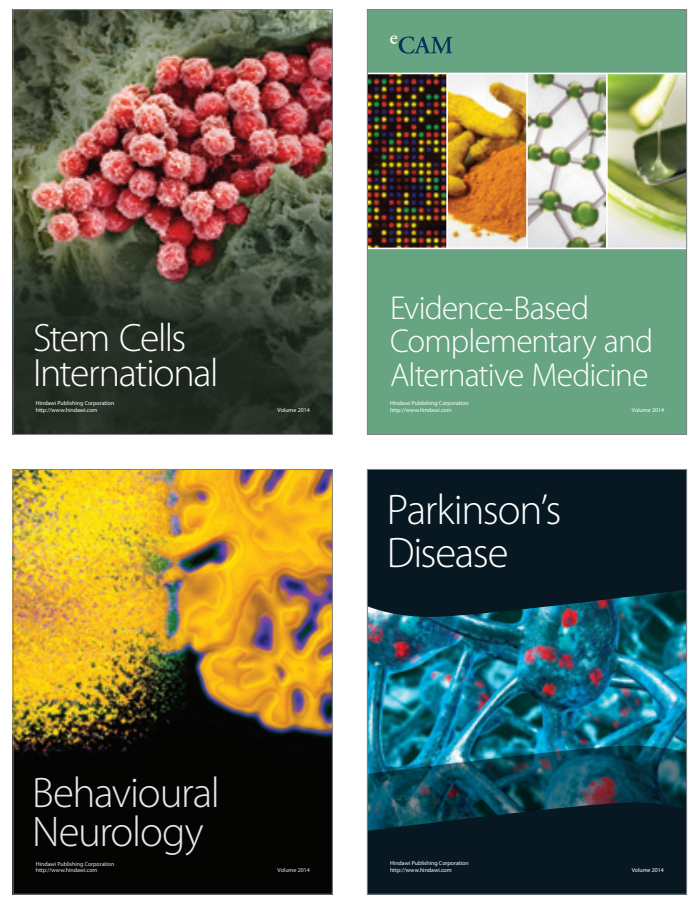
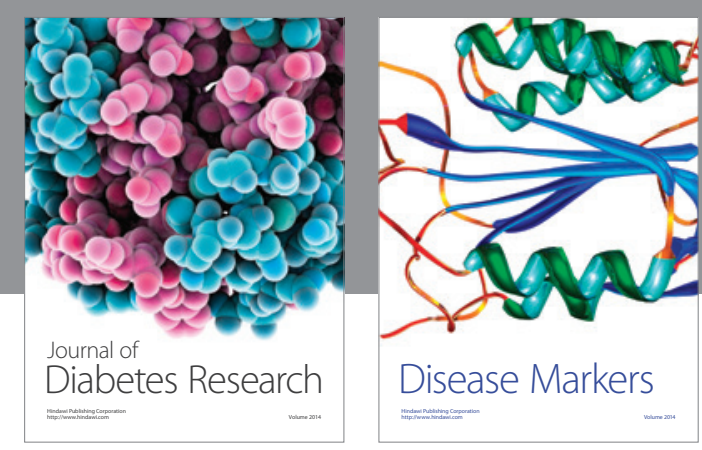

Disease Markers
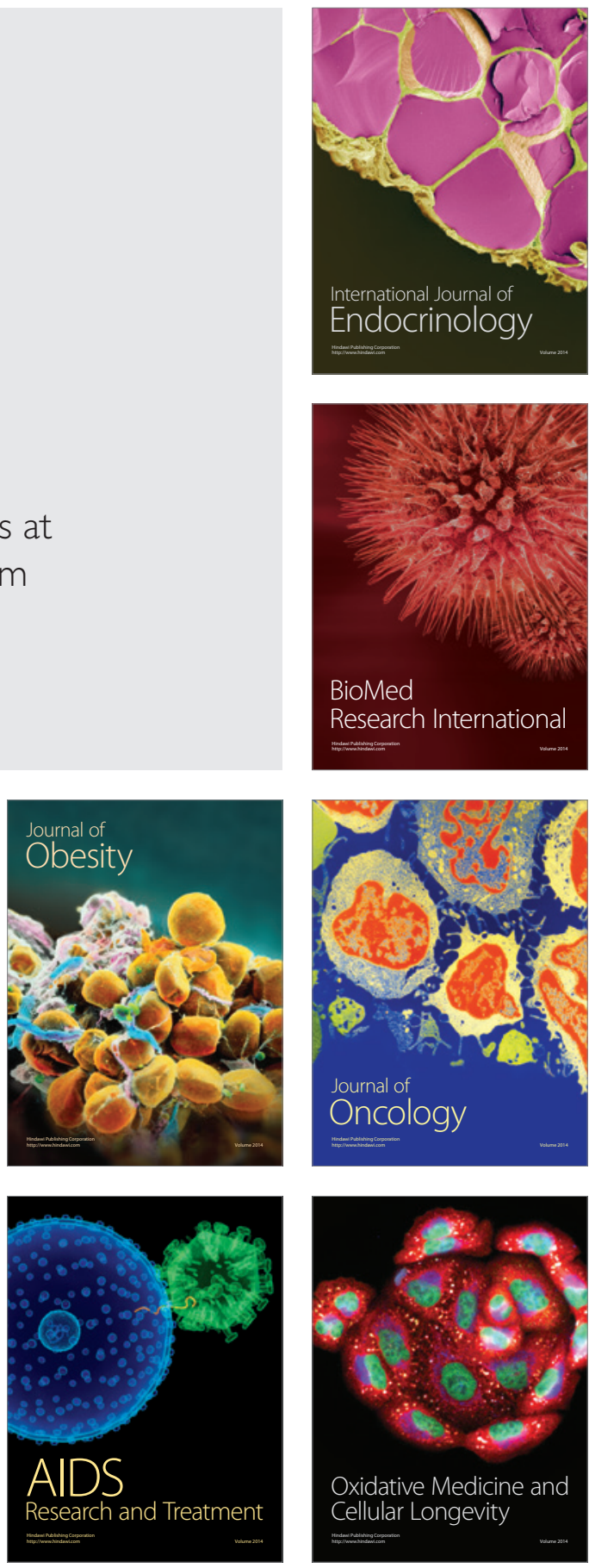